\title{
A Digital Imaging Workflow for TEM Using a Low Cost Scanner
}

\author{
J. Mackenzie, Jr.
}

Center for Electron Microscopy, North Carolina State University, Raleigh, NC 27695

Although digital imaging now dominates image acquisition in SEM, the use of digital imaging in TEM has been limited by resolution and cost. Many researchers feel pressure to remove darkrooms both for space considerations and for problems with the hazardous waste removal. For many biological applications high information density micrographs are needed. Pathology, for example, requires taking high resolution images at low magnification in order to obtain a large field of view. Although lower resolution TEM cameras can montage images, the time per image increases by a factor of four. Most of the laboratories who don't have TEM digital cameras find the TEM cameras beyond their budgets

Our current best solution is a hybrid solution that costs surprising little and yet produces exceptional results. The cost of the digital acquisition side is less than $\$ 500$ and the cost of the digital printing side is less than $\$ 500$.

The first step in our workflow is to take a standard image on conventional TEM film and process it in the normal way. Although this requires a plate processing darkroom, the size of this room can be a fraction of a printing darkroom and can be shared with others who are processing film for other scientific purposes. Although there are still chemicals that must be disposed of properly, the volume makes this option acceptable for us.

Film resolution for conventionally stained biological sections is about 10,000 pixels by 10,000 pixels at 12 bits. (10 bits?) It takes a two second exposure with an additional 30-40 seconds of plate travel time. The cost of the film and processing is about 2-3 dollars per film. The cost per film is considerably more than the virtually free cost of the digital but the resolution is in no way compromised.

The second stage in our workflow is to scan the negative in an Epson Perfection 4870 flatbed scanner. (The Epson 4990 has been announced but is not yet available.) There is no holder for the TEM film plate so the film is placed in the 4 x 5 inch holder with the 4 inch side lined up. This gives three sides of the film support. We have designed a first generation film holder and have a redesigned holder in prototyping. The standard Epson TWAIN scanning module is used with the default unsharp masking filter turned off. We normally scan in the positive transparency mode as we have found that it produces better scans than the negative mode. This is, of course, counter-intuitive but very easily confirmed. We scan in 16 bit grayscale mode in Photoshop and convert to 8 bit mode only after the original is saved.

The use of an inexpensive flatbed scanner started from a time when we were scanning prints and found the Epson scanners to have superior resolution at scan rates that were large multiples of other brands. The claimed optical resolutions soon reached a point where we began testing the quality of direct scanning of negatives. Our qualitative tests of image detail when compared to actual prints showed that the scanner was getting close to all the information we normally associated with 
conventional film prints. Other film scanners have been shown to have considerable optical distortion at the edges so we proceeded to test this scanner for distortion. We scanned a glass target (Edmund Optics, Inc.). Even at 3x magnification we could not find any measurable distortion.

We normally scan whole film plates at 1000 pixels per inch. If we want higher resolution of an area we can simply rescan an area at up to 4800 pixels per inch. There is considerable debate in the photographic community as to the resolution of film and the performance of low cost scanners. Both questions are relevant to this discussion. We have determined using a simple target (USAF 1951 resolution) that the 4870 scanner has at least 2000 pixels per inch. More comprehensive test with Modulation transfer function will be reported

The low cost of the scanner has permitted us to place one on all the desktop computers in our Center. Students can scan negatives while they perform other tasks. For our purposes, the film is the high resolution storage medium and the digital, the working medium. Once the final resolution is determined all subsequent work is digital. This hybrid solution has all the advantages of digital and never precludes us from returning to conventional photography if we have a question as to whether we would see more in a photomicrograph.
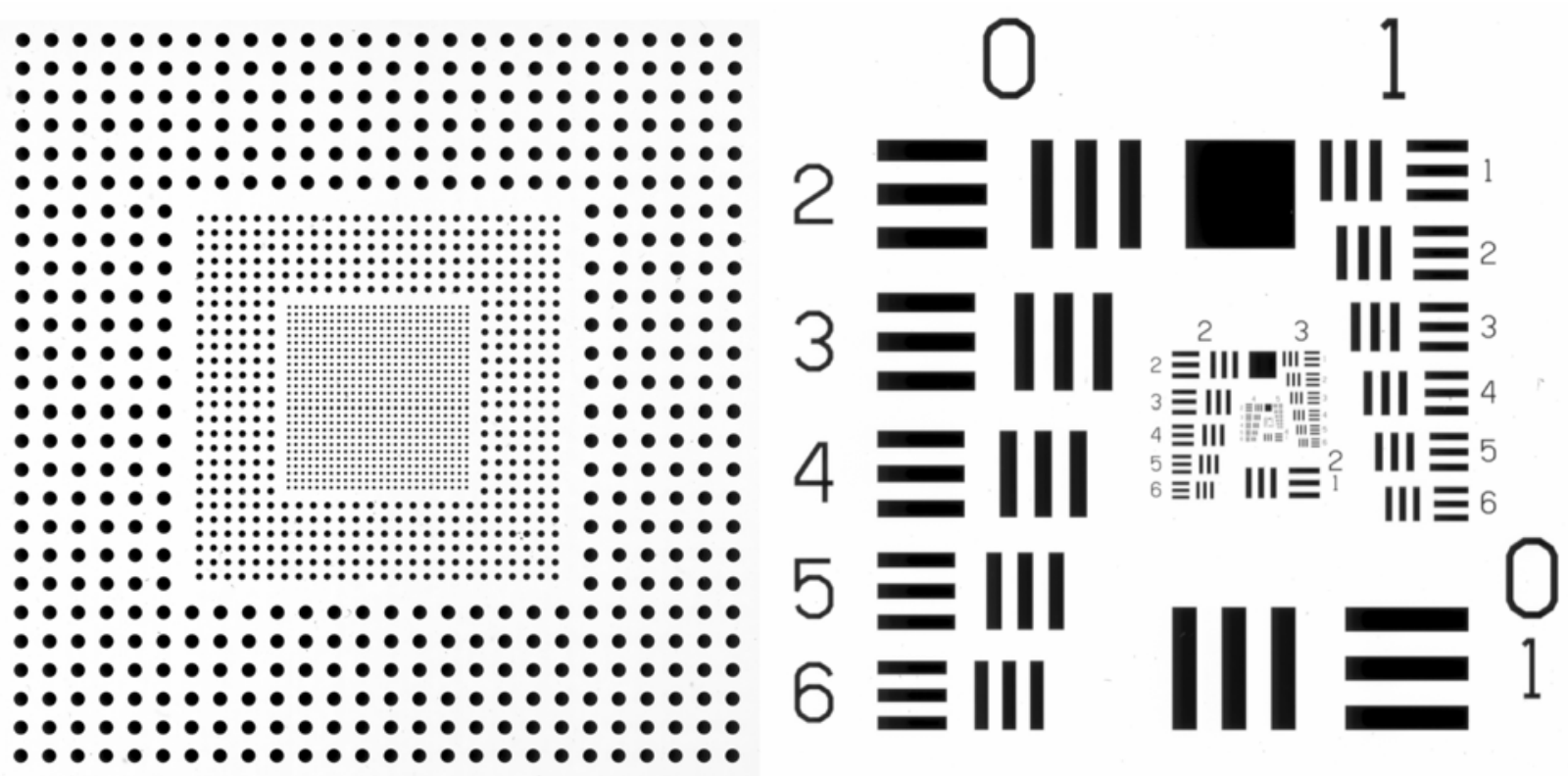

FIG. 1. Transparent test targets. Left panel is distortion target. Right panel is USAF 1951 resolution target. 